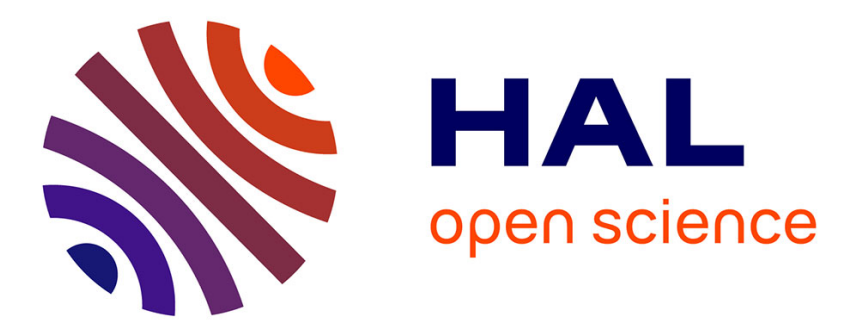

\title{
Phosphoglycerate mutase knock-out mutant Saccharomyces cerevisiae: Physiological investigation and transcriptome analysis
}

Marta Papini, Intawat Nookaew, Gionata Scalcinati, Verena Siewers, Jens Nielsen

\section{To cite this version:}

Marta Papini, Intawat Nookaew, Gionata Scalcinati, Verena Siewers, Jens Nielsen. Phosphoglycerate mutase knock-out mutant Saccharomyces cerevisiae: Physiological investigation and transcriptome analysis. Biotechnology Journal, 2010, 6 (3), pp.1016. 10.1002/biot.201000199 . hal-00573064

\section{HAL Id: hal-00573064 \\ https://hal.science/hal-00573064}

Submitted on 3 Mar 2011

HAL is a multi-disciplinary open access archive for the deposit and dissemination of scientific research documents, whether they are published or not. The documents may come from teaching and research institutions in France or abroad, or from public or private research centers.
L'archive ouverte pluridisciplinaire HAL, est destinée au dépôt et à la diffusion de documents scientifiques de niveau recherche, publiés ou non, émanant des établissements d'enseignement et de recherche français ou étrangers, des laboratoires publics ou privés. 


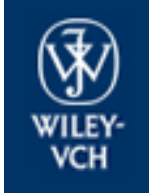

Biotechnology Journal

\section{Phosphoglycerate mutase knock-out mutant Saccharomyces cerevisiae: Physiological investigation and transcriptome analysis}

\begin{tabular}{|r|l|}
\hline Journal: & Biotechnology Journal \\
\hline Manuscript ID: & biot.201000199.R1 \\
\hline Wiley - Manuscript type: & Research Article \\
\hline Author: & 09-Jul-2010 \\
\hline Complete List of Authors: & $\begin{array}{l}\text { Papini, Marta; Chalmers University of Technology, Systems Biology } \\
\text { Nookaew, Intawat; Chalmers University of Technology, Systems } \\
\text { Biology } \\
\text { Scalcinati, Gionata; Chalmers University of Technology, Systems } \\
\text { Biology } \\
\text { Siewers, Verena; Chalmers University of Technology, Systems } \\
\text { Biology } \\
\text { Nielsen, Jens; Chalmers University of Technology, Systems Biology }\end{array}$ \\
\hline Primary Keywords: & Systems Biology \\
\hline Secondary Keywords: & Fermentation \\
\hline Keywords: & Physiology \\
\hline & \\
\hline
\end{tabular}




\section{Phosphoglycerate mutase knock-out mutant Saccharomyces} 2 cerevisiae: physiological investigation and transcriptome analysis

3 Marta Papini ${ }^{1}$, Intawat Nookaew ${ }^{1}$, Gionata Scalcinati ${ }^{1}$, Verena Siewers ${ }^{1}$ and Jens Nielsen ${ }^{* 1}$

$4 \quad{ }^{1}$ Systems Biology, Chalmers University of Technology, Chemical and Biological Engineering

5 Department, Kemigården 4, SE-41296, Göteborg, Sweden

$6 \quad{ }^{*}$ Corresponding author

$7 \quad$ Email : nielsenj@chalmers.se

$8 \quad$ Phone :+46 317723804

9

10 


\section{Abstract}

The yeast Saccharomyces cerevisiae is able to adapt its metabolism to grow on different carbon sources and to shift to non-fermentative growth on $\mathrm{C}_{2}$ or $\mathrm{C}_{3}$ carbon sources (ethanol, acetate or glycerol) through the activation of gluconeogenesis. Here we studied the response to the deletion of the glycolytic and gluconeogenic gene GPM1, encoding for phosphoglycerate mutase. It was previously shown that a $S$. cerevisiae strain with non-functional copies of GPM1 can only grow when glycerol and ethanol are both present as carbon sources, whilst addition of glucose was shown to strongly inhibit growth. It was suggested that glycerol is needed to feed gluconeogenesis whilst ethanol is required for respiration. Here we studied the physiological response of the GPM1 knock-out mutant through fermentation and transcriptome analysis. Furthermore, we compared the physiological results with those obtained through simulations using a genome-scale metabolic model, showing that glycerol is only needed in small amounts for growth. Our findings strongly indicate a severely impaired growth ability of the knock-out mutant, which presents increased transcript levels of genes involved in the pentose phosphate pathway and in the glyoxylate shunt. These results indicate an attempt to compensate for the energy imbalance caused by the deletion of the glycolytic/gluconeogenic gene within the mutant.

\section{Introduction}

The gene GPM1, located on the right arm of chromosome VIII, codes for the enzyme phosphoglycerate mutase (E.C. 5.4.2.1) which catalyzes the interconversion of 3-phosphoglycerate into 2-phosphoglycerate. In the yeast Saccharomyces cerevisiae, this enzyme is a tetramer composed of four identical subunits with a molecular weight of 28,000 Da each [1]. The gene was firstly isolated through functional complementation [2] of mutants classified among glycolytic mutants whose growth was inhibited by glucose [3, 4]. GPM1 is a constitutively expressed glycolytic gene and its promoter contains a variety of elements mediating transcriptional control [5]. Significant amounts of the enzyme are present and active also in the cell wall of yeast [6] as it was shown that phosphoglycerate mutase released from yeast cell walls upon alkaline treatment still shows enzymatic activity. Nevertheless, the role of Gpm1 in the cell wall remains largely unknown. Mutation of GPM1 in the yeast genome leads to viable cells only when glycerol and ethanol are provided together as carbon source, whilst growth of the mutant is inhibited in the presence of glucose or ethanol or glycerol alone [4]. It has been suggested that glycerol is needed to feed gluconeogenesis whereas Gibbs free energy is provided through respiration of ethanol.

Sequencing of the yeast genome has revealed two genes that have a high sequence homology to GPM1 and these have been named GPM2 and GPM3; GPM2 is positioned on the left arm of chromosome IV, whereas GPM3 is located on the left arm of chromosome XV. Heinisch et.al [7] suggested that GPM2 and GPM3 may represent non-functional homologues formed as a result of gene duplication events. Their work showed that overexpression of GPM2 or GPM3 on a multicopy vector under the control of their own promoters does not complement a gpm1 deletion mutant when grown on complex media containing glucose or a mixture of glycerol/ethanol as carbon sources. 
.

Further analysis on the genes promoters indicated that these genes are expressed at too low levels to contribute to a significant glycolytic flux. Overexpression of these two genes under the control of the PFK2 promoter can partially complement the growth defect of $g p m 1$ deletion on synthetic and complex media containing of glycerol and ethanol after prolonged incubation.

It is well known that $S$. cerevisiae is able to adapt its growth to different carbon sources by switching its metabolism. This is possible through expression of different sets of genes according to the carbon source utilized $[8,9]$. Indeed, in the transition from glucose-based fermentative growth to respiratory growth on glycerol [10] and during the diauxic shift, the expression levels of many different genes are altered. In both cases the observed physiological response is a decrease in the specific growth rate and as revealed by gene expression analysis, concomitant and similar patterns of gene expression alteration [11].

Growth on non-fermentable carbon sources such as ethanol or glycerol requires the activation of gluconeogenesis and the glyoxylate cycle $[12,13]$. Non-fermentable substrates are used to generate ATP through oxidative phosphorylation and for the generation of sugar phosphates, required by the cell for nucleotide biosynthesis, cell wall generation and for the biosynthesis of storage carbohydrates [13]. Gluconeogenic genes are regulated at the transcriptional level through the regulator Rds2 [14]; it is known that the activation of genes of gluconeogenesis depends on upstream activator sequences (UASs) found in their promoters, as well as carbon source response elements (CSREs). In absence of glucose, or at low glucose levels, the Mig1 repressor protein is inactivated through phosphorylation by Snf1. This leads to de-repression of CAT8 [15], a target of Mig1. Cat8 promotes the expression of a different set of genes, among which several gluconeogenic genes [16, 17]. Key genes that are turned on upon gluconeogenic activation are FBP1 and PCK1 [18] encoding fructose-1,6-bisphosphatase [19] and phosphoenolpyruvate carboxykinase [20] respectively, and the glyoxylate cycle genes ICL1, coding for the isocitrate lyase [21], and MLS1 [22] coding for malate synthase [23].

The aim of this work was to study the physiological response of a strain deleted in the lower part of glycolysis by deleting the gene GPM1. This modification allowed us to study how yeast responds to a non-functional gluconeogenic pathway when growing on a $\mathrm{C}_{2}$ carbon source. The deletion mutant was characterized under growth on a dual carbon source: glycerol and ethanol, and the cellular adaptations were captured by transcriptome and integrated data analysis.

\section{Materials and Methods}

\subsection{Yeast strains}

The S. cerevisiae strains used in this study were CEN.PK 113-5D (MATa SUC2 MAL2-8 ${ }^{C}$ ura3-52; kindly provided by P. Kötter, University of Frankfurt, Germany) [24] and CEN.PK 113-5D $4 g p m 1$.

\subsection{Deletion strain construction}

The gene GPM1 was deleted using a bipartite method [25]. The regions up- and downstream of the gene were amplified by PCR using primer pairs GPM-up-fw $\left(5^{\prime}\right.$ GAGGAAGCTCGCTAAATGGGATTCC-3') / GPM-up-rv 
GCAGGGATGCGGCCGCTGACCGGATTGACCGTGTCTAACTAAAACTAAC-3') and GPM-down-fw (5'-CCGATGCTAGGCGCGCCGTGCGCTGCTGTTGCCAACCAAGG-3') / GPM-down-rv (5'AAATTCACCAACCTCTCATGGCG), respectively. The $5^{\prime}$ part of the Kluyveromyces lactis KIURA3 cassette was amplified from vector pWJ1042 [26] using primers dKL-5' $\left(5^{\prime}-\right.$ GTCAGCGGCCGCATCCCTGCTTCGGCTTCATGGCAATTCCCG-3') and Int-3'(5'GAGCAATGAACCCAATAACGAAATC3'). Likewise, primers Int-5' $5^{\prime}$ ( $5^{\prime}$ CTTGACGTTCGTTCGACTGATGAGC-3') and cKI-3' $3^{\prime}$ (5'CACGGCGCGCCTAGCAGCGGTAACGCCAGGGTTTTCCCAGTCAC-3') were used to amplify the 3' part of the KIURA3 cassette. The GPM1 upstream region and the KIURA 5'part on one hand and the KIURA 3'part and the GPM1 downstream region on the other hand were then fused in a second set of PCRs using the respective outer primers for amplification. This was possible due to complementary primer extensions at the $5^{\prime}$ ends of the inner primers. Strain CEN.PK 113-5D was transformed with the two fusion PCR products, which resulted in integration at the GPM1 locus. Transformants were selected on minimal medium containing $1.7 \mathrm{gL}^{-1}$ yeast nitrogen base (YNB) without amino acids and $\left(\mathrm{NH}_{4}\right)_{2} \mathrm{SO}_{4}$ (Alfa Aesar, Karlsruhe, Germany), $5 \mathrm{gL}^{-1}\left(\mathrm{NH}_{4}\right)_{2} \mathrm{SO}_{4}, 0.77 \mathrm{gL}^{-1}$ complete supplement mixture (CSM) without uracil (MP Biomedicals, Solon, OH, USA), $2.5 \%$ (v/v) glycerol $2.5 \%(\mathrm{v} / \mathrm{v})$ ethanol and $20 \mathrm{gL}^{-1}$ agar. Direct repeats flanking the KIURA3 cassette allowed for the loop-out of the marker. Strains that had lost the marker gene were selected on minimal medium (see above) containing 50 $\mathrm{mgL}^{-1}$ uracil and $750 \mathrm{mgL}^{-1} 5$-fluoroorotic acid (5-FOA). GPM1 deletion was confirmed through PCR and sequencing of the PCR product.

\subsection{Media and growth conditions}

The cultivations have been conducted in DasGip fermentors (DasGip, Jülich, Germany) in batch mode, the two strains (reference strain and deletion mutant) were cultivated in triplicates. For all cultivations, a previously described [27] mineral salts medium was used. The medium is composed of (per liter): $\left(\mathrm{NH}_{4}\right)_{2} \mathrm{SO}_{4}, 5 \mathrm{~g} ; \mathrm{KH}_{2} \mathrm{PO}_{4}, 3 \mathrm{~g} ; \mathrm{MgSO}_{4} \bullet 7 \mathrm{H}_{2} \mathrm{O}, 0.5 \mathrm{~g}$; Antifoam 289 (A-5551, Sigma-Aldrich, St. Louis, MO, USA), $0.05 \mathrm{~mL}$; trace metals, $1 \mathrm{~mL}$ and vitamins, $1 \mathrm{~mL}$ trace metal solution. The trace metal solution consisted of (per liter): EDTA (sodium salt), $15 \mathrm{~g} ; \mathrm{ZnSO}_{4} \cdot 7 \mathrm{H}_{2} \mathrm{O}, 0.45 \mathrm{~g} ; \mathrm{MnCl}_{2} \cdot 2 \mathrm{H}_{2} \mathrm{O}, 1$ g; $\mathrm{CoCl}_{2} \cdot 6 \mathrm{H}_{2} \mathrm{O}, 0.3 \mathrm{~g} ; \mathrm{CuSO}_{4} \cdot 5 \mathrm{H}_{2} \mathrm{O}, 0.3 \mathrm{~g} ; \mathrm{Na}_{2} \mathrm{MoO}_{4} \cdot 2 \mathrm{H}_{2} \mathrm{O}, 0.4 \mathrm{~g} ; \mathrm{CaCl}_{2} \cdot 2 \mathrm{H}_{2} \mathrm{O}, 0.45 \mathrm{~g} ; \mathrm{FeSO}_{4} \cdot 7 \mathrm{H}_{2} \mathrm{O}$, $0.3 \mathrm{~g} ; \mathrm{H}_{3} \mathrm{BO}_{3}, 0.1 \mathrm{~g}$ and $\mathrm{KI}, 0.1 \mathrm{~g}$. The $\mathrm{pH}$ of the trace metal solution was adjusted to 4.0 with $2 \mathrm{M}$ $\mathrm{NaOH}$ prior to heat sterilization. The vitamin solution contained (per liter): biotin, $0.05 \mathrm{~g}$; $\mathrm{p}$-amino benzoic acid, $0.2 \mathrm{~g}$; nicotinic acid, $1 \mathrm{~g}$; Ca-pantothenate, $1 \mathrm{~g}$; pyridoxine- $\mathrm{HCl}, 1 \mathrm{~g}$; thiamine- $\mathrm{HCl}, 1 \mathrm{~g}$ and myo-inositol, $25 \mathrm{~g}$. The $\mathrm{pH}$ of the vitamin solution was adjusted to 6.5 with $2 \mathrm{M} \mathrm{NaOH}$. The vitamin solution was filter sterilized and stored at $4{ }^{\circ} \mathrm{C}$ [27]. The medium was supplemented with $2.5 \%(\mathrm{v} / \mathrm{v})$ of ethanol and $2.5 \%(\mathrm{v} / \mathrm{v})$ glycerol as carbon sources. To complement the auxotrophy of the reference strain and of the mutant uracil solution was added to a final concentration of $20 \mathrm{mgL}^{-1}$. Cells were grown in $500 \mathrm{~mL}$ baffled shake flasks in YNB medium $\left(\mathrm{NH}_{4} \mathrm{SO}_{3}, 5 \mathrm{gL}^{-1}\right.$; YNB w/o ammonia and w/o amino acids, $1.7 \mathrm{gL}^{-1}$; CSM w/o uracil, $\left.0.77 \mathrm{gL}-1\right)$ with $3 \%(\mathrm{v} / \mathrm{v})$ glycerol and $2.5 \%(\mathrm{v} / \mathrm{v})$ ethanol as carbon sources until they reached mid-exponential phase $\left(\mathrm{OD}_{600}=2\right)$. The precultures were used to inoculate the fermentors to an initial $\mathrm{OD}_{600}=0.2$. 
The batch cultures were performed in 1.0 L DasGip stirrer-pro® vessels with a working volume of 0.7 L. Agitation was maintained at $600 \mathrm{rpm}$ using a magnetic stirrer integrated in the BioBlock $\circledast$, which maintained the temperature at $30^{\circ} \mathrm{C}$. The aeration was set to $0.5 \mathrm{~L} \mathrm{~min}^{-1}$. The $\mathrm{pH}$ of the medium was maintained at 5.0 by automatic addition of $2 \mathrm{~N} \mathrm{KOH}$. The temperature, agitation, gassing, $\mathrm{pH}$ and composition of the offgas were monitored and controlled using the DasGip monitoring and control system. Dissolved oxygen was monitored with an autoclavable polarographic oxygen electrode (Mettler Toledo, Columbus, OH, USA). The effluent gas from the fermentation was analyzed for realtime determination of oxygen and $\mathrm{CO}_{2}$ concentration by DasGip fedbatch pro® gas analysis systems with the off gas analyzer GA4 based on zirconium dioxide and two-beam infrared sensor.

\subsection{Analytical methods}

Cell dry weight was measured by filtering a known volume of the culture through a pre-dried and preweighed $0.45 \mu \mathrm{m}$ pore size nitrocellulose filter (Supor®-450 Membrane Filters, PALL Life Sciences, Ann Arbor, MI, USA). The filters with the biomass were washed with water, dried for $15 \mathrm{~min}$ in a microwave oven at $150 \mathrm{~W}$ and weighed again. The optical density was determined at $600 \mathrm{~nm}$ using a Genesys 20 Spectrophotometer, Thermo Scientific, Wilmington, DE, USA).

Concentrations of glycerol, ethanol, acetate, succinate and pyruvate were analyzed by an isocratic HPLC (UltiMate ${ }^{\circledR} 3000$ Nano/Capillary Autosamplers, Dionex, Sunnyvale, CA, USA) with an Aminex HPX-87H ion exchange column (Bio-Rad, Hercules, USA) at $65^{\circ} \mathrm{C}$ using $5 \mathrm{mM} \mathrm{H}_{2} \mathrm{SO}_{4}$ as mobile phase at a flow rate of $0.6 \mathrm{~mL} \mathrm{~min}^{-1}$. Glucose, glycerol and ethanol were measured with a refraction index detector whereas succinate, acetate and pyruvate with an ultraviolet-visible light absorbance detector.

\subsection{Sampling and total RNA extraction}

For microarray analysis a $25 \mathrm{~mL}$ sample was withdrawn from the fermentor in the mid-exponential phase $\left(\mathrm{OD}_{600}=2.5\right)$. The sample was cooled immediately in ice and centrifuged at $2^{\circ} \mathrm{C}$ and $5000 \mathrm{rpm}$ for $5 \mathrm{~min}$, the supernatant discarded and the biomass immediately frozen in liquid nitrogen. The total RNA was extracted from cells through mechanical disruption with glass beads, digested with DNAse and purified using the RNeasy kit (Qiagen, Hilden, Germany). The quality of the RNA was assayed using a BioAnalyzer (Agilent Technologies, Palo Alto, CA, USA). Concentrations were determined using a NanoDrop 2000 Spectrophotometer (Thermo Scientific, Wilmington, DE, USA). $250 \mathrm{ng}$ of the total RNA was used to synthesize cDNA using Affymetrix 3' IVT Express kit and successively aRNA was synthesized (Affymetrix Inc., Santa Clara, CA, USA).

Biotinylated RNA samples were fragmented and hybridized to Affymetrix Yeast Genome Array 2.0. The Arrays were washed using an Affymetrix GenChip Fluidic station 450 and scanned using a GeneChip $\AA$ Scanner 3000 7G (Affymetrix Inc., Santa Clara, CA, USA). CEL files were generated using the Comand console software (Affymetrix). All CEL files were submitted to GEO database and have accession number GSE22122. 


\subsection{Data analysis on Microarray}

The intensity files obtained were normalized using non-linear normalization, qspline methods [28].The expression values were processed by using Probe Logarithmic Intensity Error (PLIER) [29] approach using perfect match only (pm-only) (http://www.affymetrix.com/support/technical/technotes/plier_technote.pdf). To primary evaluate the pattern of the microarray data, hierarchical clustering and statistical analysis based on Student's $t$ test using linear models together with empirical Bayes was applied [30]. The calculated p-values were corrected by multiple testing using the method of Benjamini and Hochberg [31]. Changes in gene expression with a $Q$-value (adjust-p -value) $<0.05$ were considered significant and a cutoff value of 0.05 was applied. The logarithms of $Q$-values were sorted according to the position of the genes on the chromosome and plotted in clock-wise direction as a circular plot to illustrate global response of the gene expression. $\mathrm{R}$ suite equipped with bioconductor packages was used for statistical analysis For the integrated analysis, the Q-values for each gene were mapped on the biological graphs described in the gene ontology (GO) annotation [32], genome-scale metabolic model ilN800 [33] and DNA-protein interaction network [34, 35]. Successively, the reporter algorithm [36, 37] was applied to indentify features that represented significant responses after comparison of the reference strain and mutant. Only the features that had $p$-values less than 0.001 were considered to be significant and were selected for construction of heatmaps.

\section{Results}

4.1 Physiological characterization of the deletion strain $\Delta g p m 1$ in aerobic batch cultivations on a glycerol and ethanol mixture

It was previously shown that a S. cerevisiae strain deleted in the glycolytic gene GPM1 (encoding phosphoglycerate mutase) is not able to use glucose as sole carbon source but has the capability to grow on a mixture of ethanol and glycerol [4]. Here we studied the physiological response of a $\Delta g p m 1$ strain in batch cultivations in minimal media supplemented with glycerol $(3 \% \mathrm{v} / \mathrm{v})$ and ethanol $2.5 \%$ $(\mathrm{v} / \mathrm{v})$ as carbon sources. Fig. 1 shows the growth curves of the two strains expressed both in terms of $\mathrm{OD}_{600}$ (panel $\mathrm{A}$ ) and of dry cell weight (panel $\mathrm{B}$ ); in panel $\mathrm{C}$ and $\mathrm{D}$, ethanol and glycerol consumption, respectively, are reported. Interestingly, the glycerol concentration was detected by HPLC analysis to be almost constant throughout the fermentation, showing a small decrease only for the reference strain (panel D). For this reason ethanol was considered the only substrate for yields calculations.

Table 1 reports the yields on ethanol, calculated in C-moles/C-moles, of carbon dioxide, biomass and acetate, together with specific growth rate and ethanol uptake rate. It is possible to observe that the specific growth rate ( $\mu \mathrm{max}$ ) and the specific ethanol uptake rate (rs) are lower in the $\Delta g p m 1$ mutant, indicating the heavily impaired growth phenotype of a strain deleted in the glycolytic gene. Biomass yields on substrate ( $\mathrm{Ysx}$ ) are comparable between the two strains, $\mathrm{CO}_{2}$ yields $\left(\mathrm{YsCO}_{2}\right)$ report a slightly higher value in the reference strain, whilst acetate yield (YsAc) is higher in the $\Delta g p m 1$ mutant. 
4.2 Evaluation of glycerol utilization using in silico simulations based on a yeast genome-scale metabolic model

3 As previously mentioned, the $\Delta g p m 1$ mutant did not show glycerol consumption and the reference strain showed poor uptake of glycerol. To gain insight into this behavior and to establish whether the

\subsection{Transcriptome profile and comparison analysis of the two strains}

To gain insight into the physiological behavior of the deletion mutant, its transcriptome profile was compared with that of the reference strain using Affymetrix GeneChip Yeast Genome 2.0 [38]. To assure the robustness and reproducibility of the data obtained, the starting point of our analysis was hierarchical clustering, shown in Fig. 2a. Here it is possible to observe that the three biological replicates from the reference strain (blue) do cluster separately from the three replicates of the mutant (red). The circular mapping plot of Fig. $2 \mathrm{~b}$ shows changes in gene expression levels between the reference strain (blue) and the $\Delta g p m 1$ mutant (red) where the genes are sorted according to their position on the chromosome. In this figure it can clearly be observed that a considerable number of genes are differentially expressed between the two strains. The 9 most expressed genes in the $\triangle g P m 1$ mutant are annotated, as well as GPM1 and the two GPM1 paralogs (GPM2 and GPM3). The gene GPM1, located on chromosome XI, shows the highest differential expression, being the most upregulated gene in the reference strain. Realistically, this likely signifies that its expression is absent in the $\Delta g p m 1$ mutant, serving as further indication of its successful deletion in the mutant. The paralogs 39 of GPM1, GPM2 and GPM3 have very low expression levels in the $\triangle g p m 1$ mutant. Among the most 
over-expressed ORF in $\triangle g p m 1$ mutant we found $N Q M 1$, which codes for a transaldolase of unknown function, induced upon diauxic shift and probably involved in the pentose phosphate pathway [39]. NCA3 and HSP3O are ORFs regulating expression of mitochondrial ATP synthases and HSP3O is known to be induced by ethanol treatment or heat shock. The ORF YKL107W is a putative protein of unknown function (proposed membrane protein). PIR3 is coding for a protein needed in cell wall stability, FMP45 is coding for a mitochondrial protein involved in sphingolipid pathway whereas FMP16 is a putative protein of unknown function. SIP18 is a gene involved in osmotic stress and GRE1 is a hydrophilin of unknown function involved in stress response and related to the HOG pathway. From this first view of our microarray data it emerges that the genes most involved in a response upon deletion of GPM1 are those belonging to stress family, cell wall stability and probably the pentose phosphate pathway.

In order to study the effects of GPM1 deletion on gluconeogenesis and on the pentose phosphate pathway (PPP), we first sought to investigate changes in expression levels of genes involved in the central carbon metabolism. Fig. 3a displays a simplified map of the central carbon metabolism. This map displays the genes that were differentially expressed between the reference and the mutant strain. In the figures both fold changes and Q-values are reported. Differences in fold changes are represented with different colors, whereas different thickness of the line represents different Q-values. From a global perspective, we can observe that the greatest number of differentially expressed genes are located in the gluconeogenesis, in the pentose phosphate and, to a less extent, the glyoxylate shunt pathway. The most up-regulated genes in the $\Delta g p m 1$ mutant are the gluconeogenic genes FBP1 and TDH1 (fold change above 2.5 and Q-values below 0.01 ) and the pentose phosphate pathway genes TKL1 (fold change between 2 and 2.5 and Q-value below 0.001) and GND2 (fold change above 1.2). Other gluconeogenic genes which report a significant, but lower, up-regulation are $P C K 1$, coding for the phosphoenolpyruvate carboxykinase and ENO1, coding for the enolase. Apart from the central carbon metabolism, it is possible to observe that the $\Delta g p m 1$ mutant has a significant over-expression of genes involved in the peroxisomal fatty acid $\beta$-oxidation (FOX2, POX1, SPS13, ECl1, TES1) and, to a less degree, genes involved in glycogen/trehalose catabolism. Glyoxylate cycle genes also report remarkable changes in their expression levels, particularly the malate synthase gene MLS1, isocitrate lyase genes ICL1 and ICL2 and MDH2 gene, coding for the cytoplasmic malate dehydrogenase, both involved in gluconeogenesis and the glyoxylate shunt, which show a fold change of 1.2 and $\mathrm{MDH} 2$ having the lowest Q-value.

The genes of the lower glycolysis and gluconeogenesis, especially ENO1 [40] and the glyceraldehyde3-phosphate dehydrogenase gene $T D H 1$, are also expressed at a higher level in the $\Delta g p m 1$ mutant, suggesting that this mutant attempts to increase its gluconeogenic flux. This is consistent with the observation that the two main gluconeogenic genes FBP1 and PCK1, normally present at low levels during growth on glucose [18] but showing increased expression levels upon diauxic shift, are also over-expressed in the $\triangle g p m 1$ mutant. The transcript levels of acetyl-CoA synthase gene ACS1, the acetate transporter gene $A D Y 2$ and the mitochondrial malic enzyme gene MAE1 are also slightly higher in the $\triangle g p m 1$ mutant. The pyruvate decarboxylase genes $P D C 6$ and $P D C 1$ also result over- 
expressed, suggesting a possible sink of overflow carbon to acetate. Trehalose and glycogen metabolism genes are also found to be up-regulated in the $\Delta g p m 1$ mutant.

Interestingly, the uncharacterized ORF YJLO45W is particularly up-regulated in the $\triangle g p m 1$ mutant; this ORF codes for the protein Sdh1b [41], a putative minor succinate dehydrogenase. Previous data showed that the same ORF was also overexpressed upon diauxic shift [42]. The increased expression of this ORF might link the anaplerotic production of succinate from the glyoxylate cycle in the cytosol with its transport and subsequent oxidation in the mitochondria by the TCA cycle, suggesting a possible explanation for the increased expression of glyoxylate shunt genes.

\subsection{Integrated data analysis}

In addition to the investigation the carbon central metabolism, we attempted to detect a possible response to deletion of GPM1 in areas of the metabolism not directly related to glycolysis. To gain greater understanding of the response at different levels of cellular processes, we used the microarray data to perform Gene Ontology (GO) and Transcription Factor (TF) analysis. In GO the families of genes whose changes are most important are reported, whereas TF shows increased or decreased representation of genes regulated by different transcriptor factors. Fig. 4 reports the outcome from the integrated GO (panel a) and TF (panel b) analysis. For both analyses, a p-value of 0.001 was considered to be significant and therefore chosen as cutoff value (see color key for specific $p$-values). In the reference strain, the GO analysis revealed up-regulation of genes belonging to just two families, namely ribosome biogenesis and nucleotides biosynthesis families. On the contrary, analysis of the $\Delta g p m 1$ mutant displayed a significant upregulation of a considerable number of $\mathrm{GO}$ categories. Among the most represented was the stress-response family, a large family including heat shock genes and genes encoding oxidative and membrane stress proteins; with higher $p$-values we found the $\beta$-oxidation of fatty acid family, followed by amino acids biosynthesis and growth on non-optimal carbon sources, respiratory genes, peroxisome induction pathway genes and members of the ATPase family.

The up-regulation in these families of genes is consistent with the transcription factors found to be over-represented. In particular TF analysis of the $\Delta g p m 1$ mutant also revealed increased expression in the genes regulated by transcription factors involved in stress response such as Crz1, Haa1, Hsf1, Msn2, Msn4, Skn7, Sko1, Stb5, as well as the most known key regulator transcription factors of fatty acid $\beta$-oxidation Oaf1, Pip2 and Ino2 and transcription factors acting on the respiratory capacity Mot3 and Hap4. Interestingly, also the protein Mig1 emerges among the most over-represneted transcription factors.

It is important to note that the results obtained from GO and TF analyses were consistent with each other and despite the lack of direct link between transcriptome and phenotype, the transcriptome data are in agreement with the physiological data. These findings are also supported by reporter metabolite analysis, GO Cellular compartment and molecular function analysis, for which the results are showed in Supplementary 1. 


\section{Discussion}

The transcriptome analysis performed in this study indicates that the severely impaired growth phenotype of the $\Delta g p m 1$ strain is associated with a global stress response. As it has been previously shown [3, 4], a $\Delta g p m 1$ strain is incapable of growing on glucose as the sole carbon source but growth can be restored in presence of a mixture of glycerol and ethanol (2 to $3 \% \mathrm{v} / \mathrm{v}$ ). Previous studies [1] have suggested that ethanol is respired whereas glycerol is needed to feed gluconeogenesis. Physiological data based on HPLC measurements undertaken in this study show that the $\Delta g p m 1$ mutant does not uptake glycerol whilst a low uptake can be observed in the reference strain. This observation, together with the simulation based on the genome-scale metabolic model, agrees with the hypothesis that ethanol is primarily required to fuel the TCA cycle whereas glycerol is needed, even if only in small amounts (see Table 2), for biomass formation.

The respiratory response of the $\Delta g p m 1$ strain was also analyzed. Despite $\mathrm{CO}_{2}$ yields are comparable in the reference and deletion strains (slightly higher in the reference strain), the transcriptome data shows that in the $\Delta g p m 1$ mutant there is increased expression of genes under the control of the transcription factors involved in regulation of respiration such as Hap4, main transcriptional activator and global regulator of respiratory genes and Mot3 [43]. Together with that, up-regulation of genes coding for ATPase and energy metabolism, pentose phosphate pathway and gluconeogenesis, are also observed. These observations could indicate that the mutant attempts to compensate for the energy imbalance by increasing its respiratory capacity. This hypothesis is also suggested by the respiratory quotient $R Q$ (data not shown) which shows that whilst the reference strain has fully respiratory growth, the $\Delta g p m 1$ mutant does not respire with the same efficiency.

It has been shown before [44] that adaptation to growth on ethanol requires the involvement of enzymatic activities from the glyoxylate cycle and gluconeogenesis and our results are in agreement with this. The findings that the pentose phosphate pathway and glyoxylate cycle are up-regulated in the $\triangle g p m 1$ mutant, agree with the previous observation that when respiration is not complete the TCA cycle acts as two branches [45].

A further indication of the transcriptome data is the possibility that gluconeogenesis can be fed, at least partially, by the pentose phosphate pathway, a hypothesis supported by the finding that the transketolase gene TKL1 is over-expressed and the NQM1 transladose, whose function is not yet fully elucidated, is also over-expressed.

As shown in Fig. 3, the genes involved in glycerol uptake GUT1 and GUT2 do not show increased expression in the $\triangle g p m 1$ mutant, neither do ethanol uptake genes even though transcript levels of $A L D 2$ are slightly increased. Interestingly, the genes encoding for the $P D C$ complex genes, particularly $P D C 6$ and $P D C 1$, are up-regulated in the mutant, demonstrating again that there is not a direct correlation between gene transcript levels and fluxes [46, 47].

The hypothesis that acetate secretion act as a sink for overflow carbon is supported by the physiological data showing higher acetate yield on biomass in the $\Delta g p m 1$ mutant compared to the reference strain. It has been shown before that $S$. cerevisiae cells growing on ethanol or acetate have an increased flux through the glyoxylate shunt and malic enzyme. This is in order to supply precursor 
metabolites for biomass synthesis [12] and for the production oxaloacetate that can be converted to phosphoenolpyruvate to fuel gluconeogenesis [48]. Our finding that some of the genes of PPP are over-expressed can therefore be linked to the concomitant upregulation of glyoxylate shunt genes.

The microarray analysis allowed the linking of the severe growth defect of the $\Delta g p m 1$ mutant with expression of some patterns of genes, such as the stress response family, proving to be a useful tool in analyzing biological phenotype. The growth defect of the deletion mutant is partially explained by increased expression of genes involved in ribosome biogenesis, rRNA maturation and elongation and tRNA processing in the reference strain, consistent with its faster growth. In the $\Delta g p m 1$ mutant, genes involved in glycogen degradation, such as GDB1, encoding glycogen debranching enzyme required for glycogen degradation [49], and genes of trehalose metabolism show an increase in transcript levels, with the synthase subunit of the trehalose-6-phosphate synthase/phosphatase complex TPS1 [50] reporting a log fold change of 0.524 .

As it is known that yeast cells growing under stress conditions respond by increasing glycogen catabolism and trehalose metabolism [51], these data appear to supply additional proof of the elevated stress levels in the $\Delta g p m 1$ mutant. Increased expression of genes involved in peroxisomal $\beta$-fatty acid oxidation can also be observed in the $\Delta g p m 1$ mutant, probably because of the need to supply acetylCoA to the glyoxylate pathway. The physiologically stressed phenotype of the mutant is also in agreement with Transcription Factor and Gene Ontology analysis. The majority of stress response related gene families are over-represented in the $\Delta g p m 1$ mutant and among the most represented transcription factors we find (Fig. 4b) those encoding membrane stress protein Haa1, heat shock transcription factors Hsf1 and Skn7, the more general stress response factors Msn2 and Msn4, the osmotic and oxidative stress transcription factors Sko1 and Stb5, known for its role in the oxidative stress response. Transcription Factor analysis on the $\Delta g p m 1$ mutant also showed over-representation of genes encoding transcription factors involved in $\beta$-oxidation of fatty acids, i.e. Oaf1 and Pip2, involved as well in the regulation of genes encoding peroxisomal proteins [52]. The mutant also shows derepression of phospholipid biosynthetic genes through Ino2 and up-regulation of sterol biosynthetic genes through Upc2.

As mentioned in section 4.4, an interesting outcome from the TF analysis is the finding that Mig1 is among the most significantly regulated transcription factors. Mig1 is an extensively characterized zinc finger transcriptional repressor having target genes that are under carbon catabolite repression [53]. In order to be active, Mig1 needs to be de-phosphorylated by the protein kinase Snf1, a main player in the glucose repression regulatory cascade [54]. Snf1 plays a relevant role in the cell, controlling biological processes such as glycogen, lipid and steroid metabolism, cell cycle, amino acid and protein synthesis, stress response and redox homeostatis, generation of precursors of energy together with the metabolism of alternative and non fermentable carbon sources. Mig1 can be considered as an indicator of carbon catabolite repression and the finding that Mig1 is found to be over-represented from the transcription factor analysis could indicate that the $\Delta g p m 1$ mutant is less catabolite repressed compared to the reference strain. This observation is consistent with the finding that some of the other transcription factors over-represented such as Msn2, Yap1, Oaf1 and Gcn4, Msn2 and Adr1, are also 
1 known to be related to Snf1. Our results thus point to the conclusion that even small fluxes through the 2 lower glycolysis may have a robust impact on carbon catabolite repression.

3 This study serves to highligh how transcriptome analysis can serve as valuable tool in physiological 4 studies of mutant strains, as the utilization of this technique can help to identify non-obvious parts of 5 the metabolism that are indirectly involved with the perturbation made on the biological system being 6 studied. 


\section{References}

[1] Rodicio, R., Heinisch, J., Isolation of the yeast phosphoglyceromutase gene and construction of deletion mutants. Mol Gen Genet 1987, 206, 133-140.

[2] Kawasaki, G., Fraenkel, D. G., Cloning of yeast glycolysis genes by complementation. Biochem Biophys Res Commun 1982, 108, 1107-1122.

[3] Ciriacy, M., Breitenbach, I., Physiological effects of seven different blocks in glycolysis in Saccharomyces cerevisiae. Journal of Bacteriology 1979, 139, 152-160.

[4] Lam, K., Marmur, J., Isolation and Characterization of Saccharomyces cerevisiae Glycolytic Pathway Mutants. Journal of Bacteriology 1977, 130, 746-749.

[5] Rodicio, R., Heinisch, J. J., Hollenberg, C. P., Transcriptional control of yeast phosphoglycerate mutase-encoding gene. Gene 1993, 125, 125-133.

[6] Motshwene, P., Brandt, W., Lindsey, G., Significant quantities of the glycolytic enzyme phosphoglycerate mutase are present in the cell wall of yeast Saccharomyces cerevisiae. Biochem J 2003, 369, 357-362.

[7] Heinisch, J. J., Muller, S., Schluter, E., Jacoby, J., Rodicio, R., Investigation of two yeast genes encoding putative isoenzymes of phosphoglycerate mutase. Yeast 1998, 14, 203-213.

[8] Barnett, J. A., Entian, K. D., A history of research on yeasts 9: regulation of sugar metabolism. Yeast 2005, 22, 835-894.

[9] Schuller, H. J., Transcriptional control of nonfermentative metabolism in the yeast Saccharomyces cerevisiae. Curr Genet 2003, 43, 139-160.

[10] Roberts, G. G., Hudson, A. P., Transcriptome profiling of Saccharomyces cerevisiae during a transition from fermentative to glycerol-based respiratory growth reveals extensive metabolic and structural remodeling. Mol Genet Genomics 2006, 276, 170-186.

[11] DeRisi, J. L., Iyer, V. R., Brown, P. O., Exploring the metabolic and genetic control of gene expression on a genomic scale. Science 1997, 278, 680-686.

[12] Cortassa, S., Aon, J. C., Aon, M. A., Fluxes of carbon, phosphorylation, and redox intermediates during growth of saccharomyces cerevisiae on different carbon sources. Biotechnol Bioeng 1995, 47, 193-208.

[13] Dickinson, R. a. S., M., The metabolism and molecular physiology of Saccharomyces cerevisae, 2004, 2nd edition.

[14] Soontorngun, N., Larochelle, M., Drouin, S., Robert, F., Turcotte, B., Regulation of gluconeogenesis in Saccharomyces cerevisiae is mediated by activator and repressor functions of Rds2. Mol Cell Biol 2007, 27, 7895-7905.

[15] Randez-Gil, F., Bojunga, N., Proft, M., Entian, K. D., Glucose derepression of gluconeogenic enzymes in Saccharomyces cerevisiae correlates with phosphorylation of the gene activator Cat8p. Mol Cell Biol 1997, 17, 2502-2510.

[16] Klein, C. J., Olsson, L., Nielsen, J., Glucose control in Saccharomyces cerevisiae: the role of Mig1 in metabolic functions. Microbiology 1998, 144 ( Pt 1), 13-24.

[17] Usaite, R., Jewett, M. C., Oliveira, A. P., Yates, J. R., 3rd, et al., Reconstruction of the yeast Snf1 kinase regulatory network reveals its role as a global energy regulator. Mol Syst Biol 2009, 5, 319. [18] Mercado, J. J., Smith, R., Sagliocco, F. A., Brown, A. J., Gancedo, J. M., The levels of yeast gluconeogenic mRNAs respond to environmental factors. Eur J Biochem 1994, 224, 473-481. [19] Entian, K. D., Vogel, R. F., Rose, M., Hofmann, L., Mecke, D., Isolation and primary structure of the gene encoding fructose-1,6-bisphosphatase from Saccharomyces cerevisiae. FEBS Lett 1988, 236, 195-200.

[20] Valdes-Hevia, M. D., de la Guerra, R., Gancedo, C., Isolation and characterization of the gene encoding phosphoenolpyruvate carboxykinase from Saccharomyces cerevisiae. FEBS Lett 1989, 258, 313-316. [21] Fernandez, E., Moreno, F., Rodicio, R., The ICL1 gene from Saccharomyces cerevisiae. Eur J Biochem 1992, 204, 983-990. 
[22] Hartig, A., Simon, M. M., Schuster, T., Daugherty, J. R., et al., Differentially regulated malate synthase genes participate in carbon and nitrogen metabolism of S. cerevisiae. Nucleic Acids Res 1992, 20, 5677-5686.

[23] Diaz, H., Andrews, B. A., Hayes, A., Castrillo, J., et al., Global gene expression in recombinant and non-recombinant yeast Saccharomyces cerevisiae in three different metabolic states. Biotechnol Adv 2009, 27, 1092-1117.

[24] Entian, K. D. a. K., P., Yeast Gene Analysis, in: Stark, I. S. M. J. R. (Ed.), Methods Microbiology, Academic Press Ltd., San Diego 2007, pp. 629-666.

[25] Erdeniz, N., Mortensen, U. H., Rothstein, R., Cloning-free PCR-based allele replacement methods. Genome Res 1997, 7, 1174-1183.

[26] Reid, R. J., Lisby, M., Rothstein, R., Cloning-free genome alterations in Saccharomyces cerevisiae using adaptamer-mediated PCR. Methods Enzymol 2002, 350, 258-277.

[27] Verduyn, C., Postma, E., Scheffers, W. A., Van Dijken, J. P., Effect of benzoic acid on metabolic fluxes in yeasts: a continuous-culture study on the regulation of respiration and alcoholic fermentation. Yeast 1992, 8, 501-517.

[28] Workman, C., Jensen, L. J., Jarmer, H., Berka, R., et al., A new non-linear normalization method for reducing variability in DNA microarray experiments. Genome Biol 2002, 3, research0048.

[29] Therneau, T. M., Ballman, K. V., What Does PLIER Really Do? Cancer Inform 2008, 6, 423-431. [30] Smyth, G. K., Linear models and empirical bayes methods for assessing differential expression in microarray experiments. Stat Appl Genet Mol Biol 2004, 3, Article3.

[31] Benjamini, Y., Hochberg, Y., Controlling the False Discovery Rate - a Practical and Powerful Approach to Multiple Testing. Journal of the Royal Statistical Society Series B-Methodological 1995, $57,289-300$.

[32] Hong E.L. , R. B., Qing Dong1, Karen R. Christie, Julie Park,, Gail Binkley, M. C. C., Selina S. Dwight, Stacia R. Engel,, Dianna G. Fisk, J. E. H., Benjamin C. Hitz, Cynthia J. Krieger,, Michael S. Livstone, S. R. M., Robert S. Nash, Rose Oughtred,, et al., Gene Ontology annotations at SGD: new data sources and annotation methods. Nucleic Acid Research 2008, 36, D577-581.

[33] Nookaew, I., Jewett, M. C., Meechai, A., Thammarongtham, C., et al., The genome-scale metabolic model ilN800 of Saccharomyces cerevisiae and its validation: a scaffold to query lipid metabolism. BMC Syst Biol 2008, 2, 71.

[34] Walhout, A. J., Unraveling transcription regulatory networks by protein-DNA and protein-protein interaction mapping. Genome Res 2006, 16, 1445-1454.

[35] Harbison, C. T., Gordon, D. B., Lee, T. I., Rinaldi, N. J., et al., Transcriptional regulatory code of a eukaryotic genome. Nature 2004, 431, 99-104.

[36] Patil, K. R., Nielsen, J., Uncovering transcriptional regulation of metabolism by using metabolic network topology. Proc Natl Acad Sci U S A 2005, 102, 2685-2689.

[37] Oliveira, A. P., Patil, K. R., Nielsen, J., Architecture of transcriptional regulatory circuits is knitted over the topology of bio-molecular interaction networks. BMC Syst Biol 2008, 2, 17.

[38] Wodicka, L., Dong, H., Mittmann, M., Ho, M. H., Lockhart, D. J., Genome-wide expression monitoring in Saccharomyces cerevisiae. Nat Biotechnol 1997, 15, 1359-1367.

[39] Huang, H., Rong, H., Li, X., Tong, S., et al., The crystal structure and identification of NQM1/YGR043C, a transaldolase from Saccharomyces cerevisiae. Proteins 2008, 73, 1076-1081. [40] McAlister, L., Holland, M. J., Targeted deletion of a yeast enolase structural gene. Identification and isolation of yeast enolase isozymes. J Biol Chem 1982, 257, 7181-7188.

[41] Palmieri, L., Lasorsa, F. M., De Palma, A., Palmieri, F., et al., Identification of the yeast ACR1 gene product as a succinate-fumarate transporter essential for growth on ethanol or acetate. FEBS Lett 1997, 417, 114-118.

[42] Palmieri, L., Lasorsa, F. M., Vozza, A., Agrimi, G., et al., Identification and functions of new transporters in yeast mitochondria. Biochim Biophys Acta 2000, 1459, 363-369. 
[43] Sertil, O., Kapoor, R., Cohen, B. D., Abramova, N., Lowry, C. V., Synergistic repression of anaerobic genes by Mot3 and Rox1 in Saccharomyces cerevisiae. Nucleic Acids Res 2003, 31, 58315837.

[44] de Jong-Gubbels, P., Vanrolleghem, P., Heijnen, S., van Dijken, J. P., Pronk, J. T., Regulation of carbon metabolism in chemostat cultures of Saccharomyces cerevisiae grown on mixtures of glucose and ethanol. Yeast 1995, 11, 407-418.

[45] Gombert, A. K., Moreira dos Santos, M., Christensen, B., Nielsen, J., Network identification and flux quantification in the central metabolism of Saccharomyces cerevisiae under different conditions of glucose repression. J Bacteriol 2001, 183, 1441-1451.

[46] Oliveira, J., Nielsen From gene expression to metabolic fluxes, in: Choi., S. (Ed.), Introduction to Systems Biology, Humana Press 2007.

[47] Stelling, J., Klamt, S., Bettenbrock, K., Schuster, S., Gilles, E. D., Metabolic network structure determines key aspects of functionality and regulation. Nature 2002, 420, 190-193.

[48] Chapman, K. B., Solomon, S. D., Boeke, J. D., SDH1, the gene encoding the succinate dehydrogenase flavoprotein subunit from Saccharomyces cerevisiae. Gene 1992, 118, 131-136. [49] Teste, M. A., Enjalbert, B., Parrou, J. L., Francois, J. M., The Saccharomyces cerevisiae YPR184w gene encodes the glycogen debranching enzyme. FEMS Microbiol Lett 2000, 193, 105-110.

[50] Vuorio, O. E., Kalkkinen, N., Londesborough, J., Cloning of two related genes encoding the 56$\mathrm{kDa}$ and 123-kDa subunits of trehalose synthase from the yeast Saccharomyces cerevisiae. Eur J Biochem 1993, 216, 849-861.

[51] Mahmud, S. A., Hirasawa, T., Shimizu, H., Differential importance of trehalose accumulation in Saccharomyces cerevisiae in response to various environmental stresses. J Biosci Bioeng, 109, 262266.

[52] Karpichev, I. V., Small, G. M., Global regulatory functions of Oaf1p and Pip2p (Oaf2p), transcription factors that regulate genes encoding peroxisomal proteins in Saccharomyces cerevisiae. Mol Cell Biol 1998, 18, 6560-6570.

[53] Carlson, M., Glucose repression in yeast. Curr Opin Microbiol 1999, 2, 202-207.

[54] Santangelo, G. M., Glucose signaling in Saccharomyces cerevisiae. Microbiol Mol Biol Rev 2006, 70, 253-282.

Table 1. Yields on substrate (ethanol) are reported in C-moles/C-moles with relative Standard deviation.

\begin{tabular}{|c|c|c|}
\hline Yield & Reference strain & Agpm1 \\
\hline$Y_{S X}$ & $0.442 \pm 0.02$ & $0.459 \pm 0.02$ \\
\hline$Y_{S C O}$ & $0.178 \pm 0.03$ & $0.16 \pm 0.008$ \\
\hline$Y_{S A C}$ & $0.025 \pm 0.01$ & $0.067 \pm 0.06$ \\
\hline$Y_{S S U C c}$ & $0.005 \pm 0.002$ & $0.001 \pm 0.001$ \\
\hline$\mu$ max $\left(h^{-1}\right)$ & $0.172 \pm 0.001$ & $0.097 \pm 0.009$ \\
\hline
\end{tabular}

$\mathrm{YsX}=$ biomass yield on substrate; $\mathrm{YsCO}_{2}=\mathrm{CO}_{2}$ yield on substrate; $\mathrm{YsAc}=$ acetate yield on substrate; YsSucc = succinate yield on substrate; $\mu$ max: maximum growth rate. Pyruvate is not shown due to its low values (trace amounts detected by the HPLC) 
1

2

3

4 Table 2. Theoretical glycerol uptake rate and growth rate calculated in silico based on a genome-scale

5 metabolic model for reference strain and $\Delta g p m 1$ mutant. As explained in the text, 2 different conditions 6 are analyzed.

7

\begin{tabular}{|l|l|c|c|c|}
\hline \multirow{2}{*}{ Strain } & Condition & $\begin{array}{c}\text { Growth } \\
\text { rate }\left(\mathrm{hr}^{-1}\right)\end{array}$ & $\begin{array}{c}\text { Glycerol uptake } \\
(\mathrm{mmol} / \mathrm{gDW} / \mathrm{hr})\end{array}$ & Yxgly* \\
\hline \multirow{2}{*}{ Reference } & without glycerol & 0.051 & 0 & 0 \\
\cline { 2 - 5 } & with glycerol & 0.172 & 1.298 & 0.062 \\
\hline \multirow{2}{*}{$\Delta g p m 1$} & without glycerol & 0.043 & 0 & 0 \\
\cline { 2 - 5 } & with glycerol & 0.093 & 0.580 & 0.051 \\
\hline
\end{tabular}

8

* Theoretical glycerol yield on biomass on C-moles basis

9 
1

2

3

4

5

6

7

8

9

10

11

12

13

14

15

16

17

18

19

20

21

22

23

24

25

26

27

28

29

30

31

32

33

34

35

36

37

38

39

40

41

42

43

44

45

46

47

48

49

50

51

52

53

54

55

56

57

58

59

60
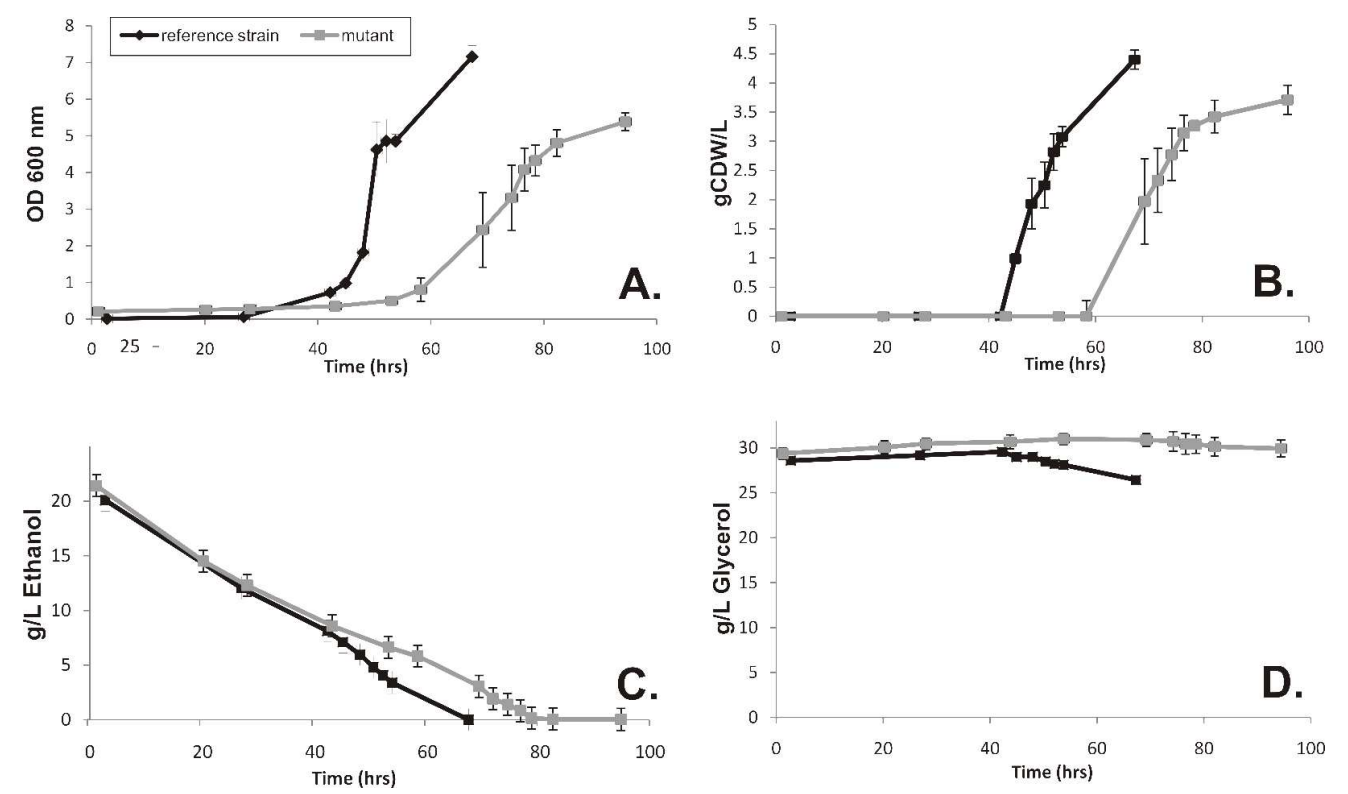

Fig. 1 Growth curve of the reference strain (black) and $\Delta g p m 1$ mutant (grey), reported as OD 600 $\mathrm{nm}$ over fermentation time (hrs) (panel A) and growth curve of the reference strain (black) and $\Delta g p m 1$ mutant (grey), reported as Dry Cell Weight (DCW) over fermentation time (panel B). For each plot, standard errors are also shown. Both the strains have been cultivated in batch mode in minimal media described in materials and methods using a mixture of glycerol and ethanol as carbon source. In panel $C$ and $D$ respectively, ethanol and glycerol consumption are reported in $\mathrm{g}^{\mathrm{L}}$ as function of fermentation time (hrs).

$205 \times 121 \mathrm{~mm}(400 \times 400$ DPI) 


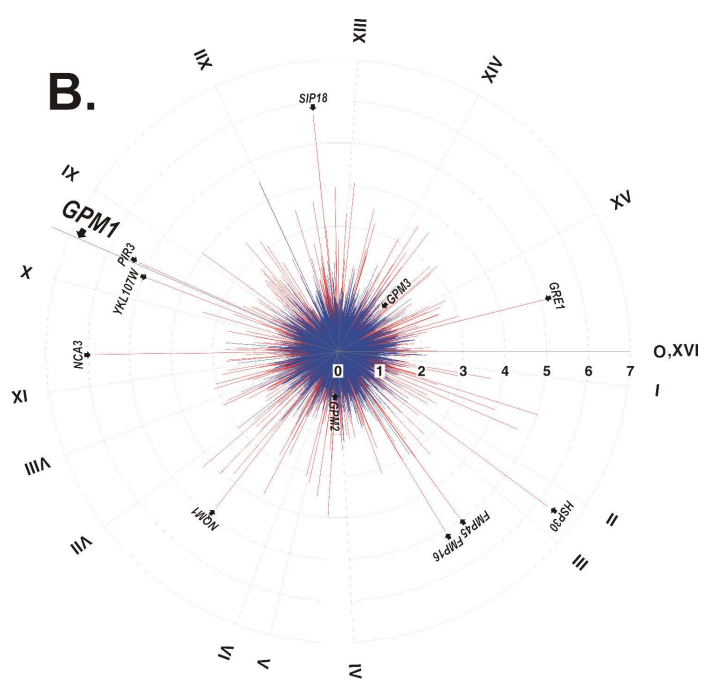

Fig. 2 Global view of gene microarray data. Panel a: The hierarchical clustering of the 6 microarray analyzed (red $=\Delta g p m 1$ mutant, blue=reference strain) shows the clustering of mutant gene expression data vs that of the reference strain, indicating the consistency of the result obtained. Panel b: Circular mapping of yeast chromosomes. The plot shows the different expression of genes between $\triangle g p m 1$ mutant (red) and the reference strain (blue) according to chromosome position. Qvalues are sorted by gene order along the chromosomes and plotted in clockwise direction. $177 \times 90 \mathrm{~mm}(400 \times 400$ DPI $)$ 

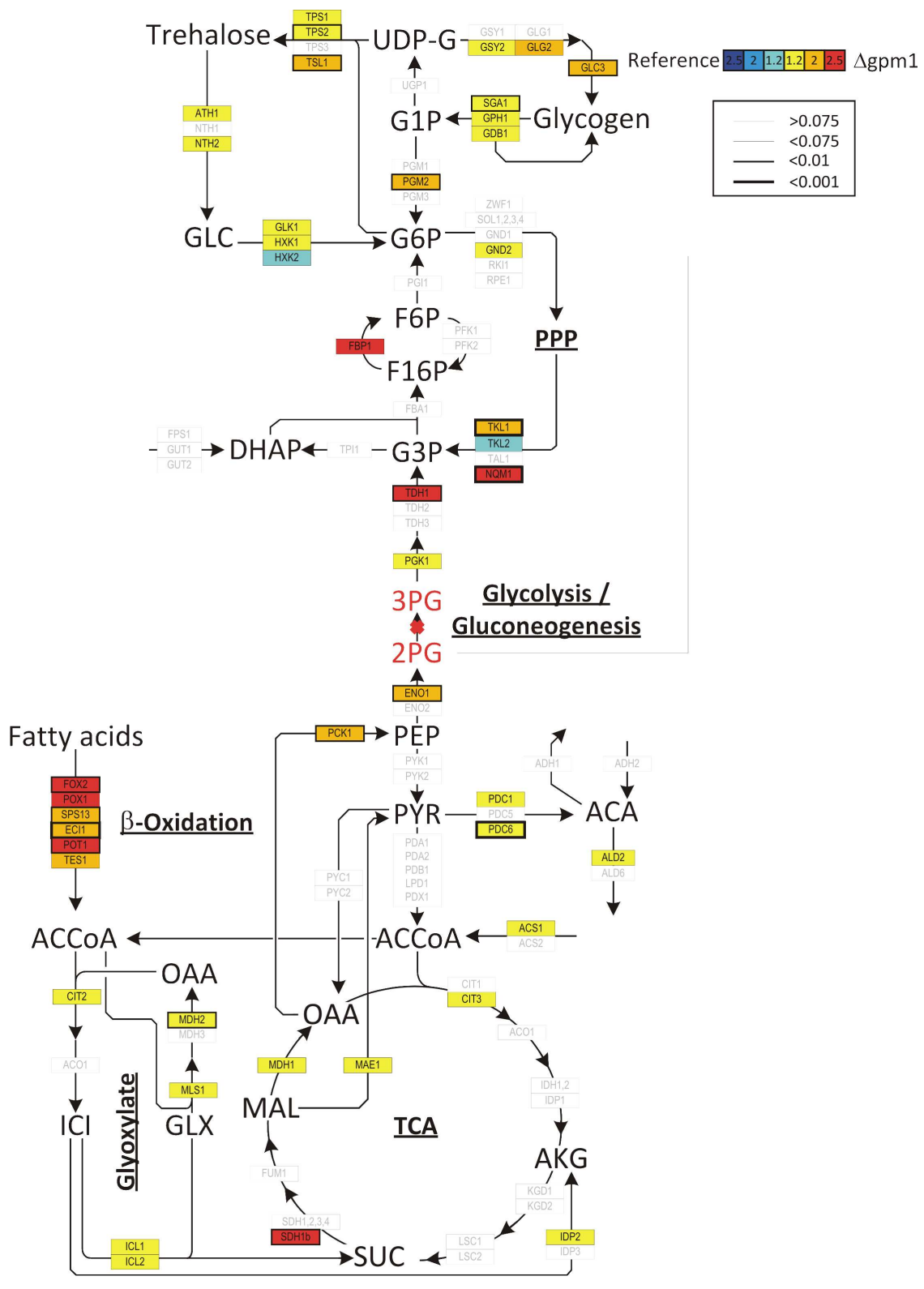

Fig. 3 Representation of gene expression levels in the yeast central carbon metabolism. Different color (see legend) expresses differences in fold changes (red= more expressed in the $\Delta g p m 1$ mutant, blue $=$ more expressed in the reference strain) whereas different thickness of the line represent Q-values (the most thick shows high significant based on Q-values). $142 \times 198 \mathrm{~mm}(400 \times 400 \mathrm{DPI})$ 
A. GO:Biological Process

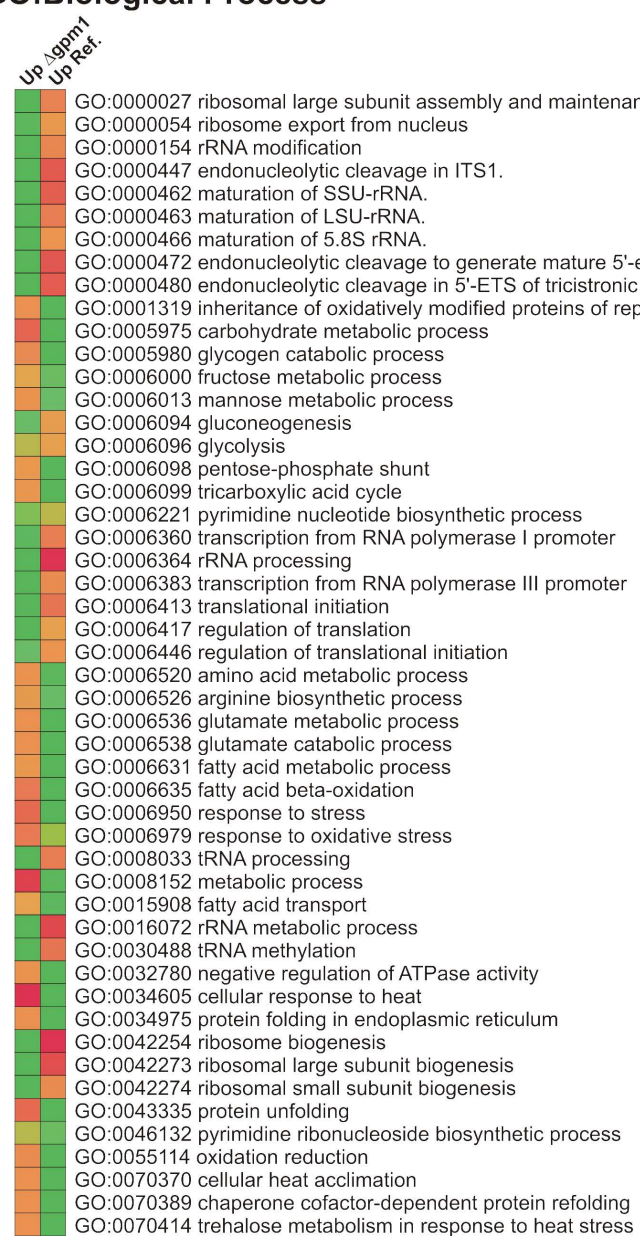

B. Transcription factor

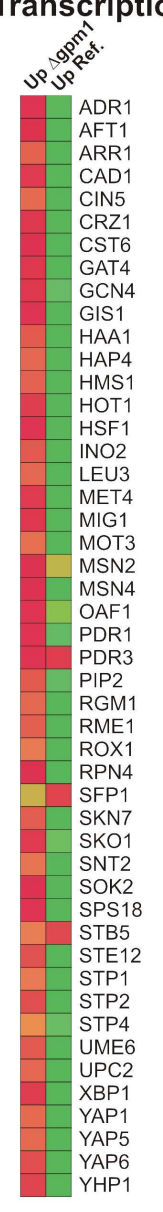

Fig.4 Integrated analysis of Gene Ontology Biological process (panel a) and Transcription Factors analysis (panel $b$ ). The different color indicates different $p$-values. All = all genes which are in the feature were considered; $\Delta g p m 1$ mutant= only over-expressed genes in the $\Delta g p m 1$ mutant were considered; ref = only genes over-expressed in the reference strain were considered. $173 \times 168 \mathrm{~mm}(400 \times 400 \mathrm{DPI})$ 